\title{
Selective Lateral Pelvic Lymph Dissection for Rectal Cancer
}

\author{
George J. Chang, MD, MS ${ }^{1,2}$ \\ ${ }^{1}$ Section of Colon and Rectal Surgery, Department of Surgical Oncology, The University of Texas, MD Anderson Cancer \\ Center, Houston, TX; ${ }^{2}$ Department of Health Services Research, The University of Texas, MD Anderson Cancer Center, \\ Houston, TX
}

While the approach to curative treatment of low rectal cancer in the West has included preoperative radiation therapy (RT) followed by total mesorectal excision (TME), in Japan the "gold standard" traditionally has been TME with prophylactic dissection of the iliac and obturator lymph nodes without pelvic radiotherapy. ${ }^{1,2}$ The divergent philosophy in the approach to curative management of pelvic micrometastases was most recently highlighted by the JCOG 0212 trial, which evaluated the noninferiority of TME alone to TME with routine prophylactic lateral pelvic lymph node dissection (LPLND) among patients without clinical evidence of lateral pelvic lymph node metastasis. ${ }^{3}$ In this study, no patients received radiotherapy and the experimental treatment strategy of TME alone was inferior to the control strategy of TME + LPLND for local control. JCOG 0212 also demonstrated that lateral pelvic lymph node metastases occurred in $7 \%$ of patients who were radiographically nodenegative within the lateral compartment before surgery and that the addition of LPLND resulted in a 5.2\% absolute reduction in the rate of local failure. ${ }^{4}$

While in the experienced hands of the JCOG 0212 investigators and in Japanese patients, the addition of LPLND had a limited effect on the overall rate of postoperative complications ( $22 \%$ vs. $16 \%$ grade $3-4$ for TME + LPLND vs. TME alone, respectively), it did add significantly to the length of operation and to total blood loss. Furthermore, the results of JCOG 0212 would suggest that $93 \%$ of patients may have undergone LPLND unnecessarily.

(C) Society of Surgical Oncology 2017

First Received: 19 August 2017;

Published Online: 27 September 2017

G. J. Chang, MD, MS

e-mail: GChang@mdanderson.org
In this issue of Annals of Surgical Oncology, Miyake and colleagues report the use of a one-step nucleic acid amplification (OSNA) strategy to aid in the selection of patients at risk for lateral pelvic lymph node metastasis, who are candidates for prophylactic LPLND. The OSNA assay semiquantitatively detects the mRNA of cytokeratin 19 (CK19) and was developed for the purpose of rapidly identifying sentinel lymph node (SLN) metastasis during breast cancer surgery without the need for a pathologist to perform frozen-section evaluation. The strategy of identifying SLN metastases plays a critical role in operative decision making for patients with breast cancer; however, the utility of such a strategy has not been established in colorectal cancer, in part because mesenteric lymphadenectomy is still an obligatory part of colorectal cancer resection and adds no significant morbidity to the primary bowel resection. However, the addition of LPLND is not an obligatory procedure and itself is associated with increased operative time, blood loss, and potential for morbidity. Thus, there is a significant appeal to identify an easily performed, rapid intraoperative assessment to guide decision-making.

While the OSNA assay may lack specificity, in this study it was highly sensitive with a high negative predictive value, even after preoperative chemotherapy. An OSNA-guided strategy could have avoided the need for prophylactic LPLND in 18 of 25 (72\%) patients, and all 4 $(16 \%)$ of the lateral pelvic lymph node-positive patients would have undergone dissection. However, whether such an approach could be broadly applied in other centers within Japan, let alone outside of Japan is unknown. The investigators identified a median of 40 lymph nodes within the mesorectum of the resected specimens. The resected rectum would have to be delivered to the pathology laboratory. All of the lymph nodes would have to be picked out (in Japan, by a member of the surgical team) and then cut 
and subject to the OSNA assay, all during the midst of the operation.

The Western and Japanese concepts are philosophically different. One relies on the tumoricidal effect of radiotherapy on potential micrometastatic spread, including along lymphatic, perivascular, and perineural pathways. The other focuses on surgical removal of lymph nodes as the primary target. However, the certainty of complete eradication of tumor cells by radiation can be no more assured than the complete resection of micrometastases along residual lymphatic drainage channels within the pelvis with LPLND. Perhaps this explains why local recurrence can occur despite either preoperative chemoradiation therapy with TME alone or without preoperative chemoradiation therapy and TME with prophylactic LPLND. In JCOG 0212, the local failure rate among patient who underwent TME + LPLND was still $7.4 \%$, which is not very different from the results achieved with TME + preoperative radiotherapy. ${ }^{5}$ In fact, in the MRC CR07 trial, the 3-year rate of local recurrence among the higher risk stage III patients also was $7.4 \%$.

Increasingly, rectal surgeons in Japan and the West have begun to move beyond an EAST-WEST debate. Advances in high-resolution imaging technology can identify lateral compartment lymph nodes that are at risk for tumor involvement. Chemoradiation therapy without LPLND among patients with lateral compartment lymph nodes $>10$ $\mathrm{mm}$ on short axis risks local failures in $33.3 \% .{ }^{6}$ However, when followed by selective LPLND for patients with lymph nodes greater than 7-8 $\mathrm{mm}$ is associated with a low risk $(0.9-3.4 \%)$ of lateral compartment failure with an incidence of pathologically confirmed persistent lateral node involvement in more than half the dissected patients. ${ }^{7,8}$ Although the jury has not determined whether the decision of dissection may be made after evaluation of the posttreatment response, a selective LPLND strategy has the potential to identify the few patients who may benefit from LPLND and safely avoid it in nearly all other patients.
The optimal management strategy for patients with low rectal cancer continues to be debated. However, it is increasingly clear that whether the primary treatment strategy incorporates selective radiotherapy or selective lateral node dissection, there are patients who may need both. While the concept of an intraoperative assessment to determine the extent of surgery is an appealing one, a practical, broadly usable method so remains to be identified. Miyake and colleagues are to be congratulated on trying to move the field forward in the direction of that goal.

\section{REFERENCES}

1. Benson AB 3rd, Venook AP, Bekaii-Saab T, et al. Rectal cancer, Version 2.2015. J Natl Compr Canc Netw. 2015;13:719-28.

2. Watanabe T, Muro K, Ajioka Y, et al. Japanese Society for Cancer of the Colon and Rectum (JSCCR) guidelines 2016 for the treatment of colorectal cancer. Int J Clin Oncol. 2017. doi:10. 1007/s10147-017-1101-6.

3. Fujita S, Akasu T, Mizusawa J, et al. Postoperative morbidity and mortality after mesorectal excision with and without lateral lymph node dissection for clinical stage II or stage III lower rectal cancer (JCOG0212): results from a multicentre, randomised controlled, non-inferiority trial. Lancet Oncol. 2012;13:616-21.

4. Fujita S, Mizusawa J, Kanemitsu Y, et al. Mesorectal excision with or without lateral lymph node dissection for clinical Stage II/III lower rectal cancer (JCOG0212): a multicenter, randomized controlled, noninferiority trial. Ann Surg. 2017;266:201-7.

5. Sebag-Montefiore D, Stephens RJ, Steele R, et al: Preoperative radiotherapy versus selective postoperative chemoradiotherapy in patients with rectal cancer (MRC CR07 and NCIC-CTG C016): a multicentre, randomised trial. Lancet. 2009;373:811-20.

6. Kusters M, Slater A, Muirhead R, et al. What to do with lateral nodal disease in low locally advanced rectal cancer? A call for further reflection and research. Dis Colon Rectum. 2017;60:577-85.

7. Akiyoshi T, Ueno M, Matsueda K, et al. Selective lateral pelvic lymph node dissection in patients with advanced low rectal cancer treated with preoperative chemoradiotherapy based on pretreatment imaging. Ann Surg Oncol. 2014;21:189-96.

8. Ishihara S, Kawai K, Tanaka T, et al. Oncological outcomes of lateral pelvic lymph node metastasis in rectal cancer treated with preoperative chemoradiotherapy. Dis Colon Rectum. 2017;60:469-76. 